\title{
Artificial Intelligence in Dental Medicine
}

\section{Anita Mehta ${ }^{*}$, Isham Mittal ${ }^{2}$ and Anushka Kakkar ${ }^{3}$}

${ }^{1}$ Professor, Department of Periodontology and Implantology, Dasmesh Institute of

Research and Dental Sciences, Affiliated with Baba Farid University of Health

Sciences, Faridkot, Punjab, India

${ }^{2}$ Government Dental College, Amritsar, India

${ }^{3}$ Professor, Guru Gobind Singh Medical College, Affiliated with Baba Farid

University of Health Sciences, Faridkot, Punjab, India

*Corresponding Author: Anita Mehta, Professor, Department of Periodontology

Baba Farid University of Health Sciences, Faridkot, Punjab, India.

Received: November 25, 2021

Published: January 07, 2022

(C) All rights are reserved by Anita Mehta.,

et al.
Artificial intelligence means machines that perform and function work which are similar to those which are performed by humans. Artificial intelligence initially started after the Second World War and has great scepticism, interest and disinterest [1]. Recently, artificial intelligence due to advances in computer technology has attracted new attention. Machine learning is artificial intelligence which uses algorithms to know the patterns in data sets and so predict these patterns in other data. Deep learning is a part of machine learning that with its neural networks performs the functions of the human brain.

We see evidence of artificial intelligence in our daily lives. Artificial intelligence is at work, when we make on-line purchase and then we receive their follow-up messages that offers similar products with discount. In the field of healthcare, artificial intelligence is applied. In many clinical fields, artificial intelligence occurred like in medicine, ophthalmology and diagnostic radiology.

\section{Ophthalmology}

Artificial intelligence applied in the identification of many eye disorders which includes glaucoma, diabetic retinopathy, age-related macular degeneration [3]. Diabetes has many complications like diabetic retinopathy and approximately one-third of diabetic patients can develop this complication. Many techniques are there that can visualize at the back of the eye for Diabetic retinopathy. For early detection of diabetic retinopathy, screening is very im- portant tool. The performance of Artificial intelligence/deep learning systems to detect Diabetic retinopathy has been shown to be very good. There is $87 \%$ to $100 \%$, sensitivity (the percent of positive cases that are identified) and $73 \%$ to $98 \%$ of specificity (the percentage of negative cases that are identified).

In resource poor countries and in remote areas where an ophthalmologist is not available these cost-saving techniques used to screen diabetic patients. In case of telemedicine for diagnosis and for initiation of treatment, these techniques can be linked.

\section{Diagnostic radiology}

Artificial intelligence can be used for examination of medical imaging. For identification of the complex patterns which has many medical images, artificial intelligence systems can provide quantitative analysis of these patterns with the intent of identifying abnormalities. For example, application of chest x-ray is artificial intelligence. Radiologists in different institutions may not agree as closely [5]. Identification of COVID-19 pneumonia is also an example. Reverse transcription polymerase chain verified COVID-19 infection). The COVID-19 pneumonia on radiographs was identified approximately $80 \%$.

\section{Application of artificial intelligence in dentistry}

According to Park and Park [9] in healthcare, artificial intelligence in different fields aids in decision-making by allowing non- 
specialists to function as specialists. Artificial intelligence applied in healthcare disciplines, in identification and diagnosis of risk and outcomes. In different dental specialties these include.

\section{Orthodontics}

- Decision-making in the need for tooth extraction.

- Unerupted tooth size prediction.

- Management of impacted canine.

- In cephalometric diagnosis.

- Skeletal malocclusion production which is related to mandibular morphology.

Restorative dentistry and prosthodontics

- In predicting restoration longevity.

- For esthetic restoration: color matching.

- Removable partial dentures design.

- In tooth-whitening methods: predicting the outcome.

Periodontology and oral medicine and radiology

- Periodontal disease: diagnosis and classification.

- In the use of immunologic parameters to diagnose aggressive periodontitis.

- Halitosis: classification based on identification of periodontal pathogens in saliva.

- In predicting recurrence of aphthous ulcers.

Temporomandibular joint disorders

- To determine the prognosis of TMJ disorders: use of magnetic resonance imaging (MRI).

- In treatment of TMJ disorders: use of screening questions to aid.

- In TMJ disorders: identification of subgroups of internal derangement.

\section{Endodontics}

- For accessory canals location.

- In detection of periapical radiolucencies [11].

Oral and maxillofacial surgery

- In the root fractures detection.

- Dental implants treatment: in the clinical decision.
Oral cancer

- For prognosis of oral cancer by considering the lesion histology and genetic characteristics.

- Following treatment of oral/oropharyngeal cancer: hypernasal speech treatment.

- Oral cancer: risk assessment.

In clinical dentistry, in research studies, in oral and maxillofacial radiology, artificial intelligence is applied. Caries and periodontal diseases are the most common dental diseases that occur all over the world.

Artificial intelligence for dental imaging suggests that the most common uses for artificial intelligence in dental radiology will be for identification of cysts and tumors of the jaws, identification of osteoporosis, cephalometric analysis and identification of periapical and periodontal infection [12].

Artificial intelligence will occur with interpretation of two-dimensional radiographs, in clinical dentistry. These will scan radiographs, identify anatomy and pathologic findings., This technology also enhances quality control across the multiple offices from the large organization's perspective. To assure all documentation, dental insurance companies can also utilize this technology. There is application of artificial intelligence in CBCT expansion. Artificial intelligence use will certainly improve clinical accuracy and efficiency.

The introduction of Artificial intelligence in dentistry is now gaining momentum, especially in these times of re-imagining clinical care and considering new ways to engage patients. Artificial intelligence systems for radiographic interpretation in dental offices will provide the following:

- The identification of deviations from normal, specifically dental and osseous pathology.

- In greater ease of tracking changes in radiographs over time.

- $\quad$ For Improved efficiency within the practice.

- In this Integration of medical and dental practices will be enhanced. Conversely, medical providers can have access to information in the dental record that may impact chronic disease, i.e., severity of periodontal disease.

- For Improved patient relations as the introduction of these systems will build patient/provider trust.

Citation: Anita Mehta., et al. "Artificial Intelligence in Dental Medicine". Acta Scientific Dental Sciences 6.2 (2022): 57-59. 
- In the introduction of these systems will differentiate practices, with a focus on adoption of new technology.

- If Potential exists for using these systems to help educate students and younger members of the profession in radiographic interpretation.

In a time-efficient manner, these systems should improve the quality of care.

\section{Bibliography}

1. Horgan J. "Will artificial intelligence ever live up to its hype?" Scientific American (2020).

2. Schwendicke F., et al. "Artificial intelligence in dentistry: Chances and challenges". Journal of Dental Research 99.7 (2020): 769-774.

3. Ting DSW., et al. "Artificial intelligence and deep learning in ophthalmology". British Journal of Ophthalmology 103.2 (2019): 167-175.

4. Hosny A., et al. "Artificial intelligence in radiology". Nature Reviews Cancer 18.8 (2018): 500-510.

5. Yasaka $\mathrm{K}$ and Abe O. "Deep learning and artificial intelligence in radiology: Current applications and future directions". PLOS Medicine 15.11 (2018): e1002707.

6. Murphy K., et al. "Covid-19 on chest radiographs: A multireader evaluation of an artificial intelligence system". Radiology 296.3 (2020): E166-E72.

7. Mintz Y and Brodie R. "Introduction to artificial intelligence in medicine". Minimally Invasive Therapy and Allied Technologies 28.2 (2019): 73-81.

8. Rajkomar A., et al. "Machine learning in medicine". The New England Journal of Medicine 380.14 (2019): 1347-1358.

9. Park WJ and Park JB. "History and application of artificial neural networks in dentistry". European Journal of Dentistry 12.4 (2018): 594-601.

10. Machoy ME., et al. "The ways of using machine learning in dentistry". Advances in Clinical and Experimental Medicine 29.3 (2020): 375-384.

11. Endres MG., et al. "Development of a deep learning algorithm for periapical disease detection in dental radiographs". Diagnostics (Basel) 10.6 (2020).
12. Hung K., et al. "The use and performance of artificial intelligence applications in dental and maxillofacial radiology: A systematic review". Dentomaxillofacial Radiology 49.1 (2020): 20190107.

\section{Assets from publication with us}

- Prompt Acknowledgement after receiving the article

- Thorough Double blinded peer review

- Rapid Publication

- Issue of Publication Certificate

- High visibility of your Published work

Website: www.actascientific.com/

Submit Article: www.actascientific.com/submission.php Email us: editor@actascientific.com

Contact us: +919182824667 